\title{
ANALYSIS OF THE ANTIOXIDANT ACTIVITY OF GERANIOL EMPLOYING VARIOUS IN-VITRO MODELS: RELEVANCE TO NEURODEGENERATION IN DIABETIC NEUROPATHY
}

\author{
SATHYA N PRASAD ${ }^{1,2 *}$, MURALI MURALIDHARA ${ }^{2}$ \\ ${ }^{1}$ Department of Biochemistry, School of Life Sciences, Vels University, Chennai, Tamil Nadu, India. ${ }^{2}$ Department of Biochemistry and \\ Nutrition, CSIR, Central Food Technological Research Institute, Mysuru, Karnataka, India. Email: sathya1prasad@gmail.com
}

Received: 19 March 2017, Revised and Accepted: 14 April 2017

\section{ABSTRACT}

Objective: The aim of this study was to analyze antioxidant effect of geraniol (GE) in different in vitro models.

Methods: Initially, the antioxidant activity of GE was assessed by diphenyl picrylhydrazyl radical (DPPH) assay. The modulatory effect of GE against 2,2'-azobis(2-amidinopropane) dihydrochloride induced lipid peroxidation in rat brain regions (cortex and cerebellum) and sciatic nerve (SN) homogenates was determined. Further, the effect of GE was assessed against hyperglycemia-induced oxidative stress (OS) in SHSY5Y, a human neuroblastoma cell line.

Results: GE proved to be a good scavenger of DPPH free radical (inhibitory concentration $50 \%$ [IC ${ }_{50}$ ] value $=663 \mathrm{nmol}$ ) and could lower the lipid peroxidation levels in rat brain tissue and SN homogenates (25-40\%). Further, it rescued the SHSY5Y cells from hyperglycemia-induced death. Coexposure of $\mathrm{GE}$ with the $\mathrm{IC}_{50}$ level of glucose $(100 \mathrm{mM})$ lowered the levels of reactive oxygen species, hydrogen peroxides and 3-nitrotyrosine levels with concomitant elevation in the glutathione levels (about two folds).

Conclusion: Collectively from these findings and other studies previously conducted (from our lab and others) emphasize the potential benefit of GE against oxidative stress (OS), a progressive pathological feature of neurodegenerative disorders.

Keywords: Geraniol, Antioxidant, In vitro studies, Diabetic neuropathy.

(c) 2017 The Authors. Published by Innovare Academic Sciences Pvt Ltd. This is an open access article under the CC BY license (http://creativecommons. org/licenses/by/4. 0/) DOI: http://dx.doi.org/10.22159/ajpcr.2017.v10i7.18564

\section{INTRODUCTION}

Oxidative stress (OS) has been understood as one of the prominent features leading to the progression of diabetic neuropathy (DN) and other neurodegenerative disorders (NDD) [1,2]. Development of higher levels of reactive oxygen species (ROS) and reactive nitrogenous species (RNS) is evident in the nervous tissue in various NDD such as Alzheimer's disease (AD) and Parkinson's disease (PD). These reactive species include hydroxyl radical, superoxide, nitric oxide, hydrogen peroxide, and peroxynitrites. Overproduction of ROS/RNS, with a concomitant, compromised antioxidant defense system results in significant protein oxidation, lipid peroxidation and nucleic acid oxidation [1]. These oxidized molecules with hampered structure/function lead to various cellular processes including inflammatory response, mitochondrial dysfunction, and possibly apoptosis in NDD [2-4]. DN is a common neurodegenerative complication with no definite pharmacological solution because of its multifactorial etiology [5].

In this scenario, plant-derived compounds with multiple target might play a role in drug discovery and development. A number of studies have demonstrated potential health-promoting properties of natural products as therapeutics for NDD [6-8]. Several epidemiological reports have documented the influence of dietary habits on lower incidence of NDD. In particular, a significant positive correlation between the consumption of polyphenolic phytochemical-rich foods and the prevention of certain neurological diseases, has been emphasized. Many bioactives such as eugenol, bacopasides, withanolide, and curcumin have been investigated for their neuroprotective function in various NDD models [9-11].

Geraniol (GE) is a bioactive belonging to the class of monoterpenoids [12]. It is found as an important constituent of essential oils of various aromatic herbs. It has a pleasant aroma and is characteristic of rose oil and citrus fruits [12,13]. It is also found in differential amounts in several herbs such as coriander and allspice. It has applications due to its aroma in food and beverage industries as a flavoring agent. It is also found in a wide range of cleansing products and cosmetics for its appealing odor [13,14]. In the recent past, several studies have recognized the pharmacological potential of GE in different models [15-17]. Its pharmacological property is by virtue of its antioxidant and anti-inflammatory properties $[18,19]$.

However, phytochemicals in general and polyphenols, in particular, can also exert pro-oxidant activities under certain experimental conditions [20]. To date, neither pro-oxidant nor antioxidant activities have yet been clearly established to occur in humans. The mechanism through which such dietary supplementation may diminish deleterious effects taking place during degenerative processes of a given disease is not clearly understood. In this study, an attempt has been made to investigate the antioxidant potential of GE in brain and sciatic nerve (SN) homogenates. This was followed by the investigation of the modulatory potency of GE in SHSY5Y, neuroblastoma cell line, under hyperglycemic condition simulating DN. These studies were designed based on the data pertaining to the modulatory effects of GE obtained in in vivo studies from the same lab earlier $[16,21]$.

\section{METHODS}

GE, diphenyl picrylhydrazyl radical (DPPH), 2,2'-Azobis(2amidinopropane) dihydrochloride (AAPH), KRBS reagent, thiobarbituric acid (TBA), trichloroacetic acid, glacial acetic acid, sodium nitroprusside, Griess reagent, hydrogen peroxide, Lowry's reagent, and FC reagent were purchased from Aldrich-Sigma and SRL. All other chemicals and solvents were of analytical grade and were purchased from local suppliers of manufacturers such as Merck, Himedia, Sigma and SRL. 
Free radical scavenging potency of GE in chemical systems

The antioxidant potential of GE was assessed employing following in vitro methods as described earlier [9].

\section{DPPH radical scavenging assay}

The DPPH free radical scavenging assay was performed by incubating aliquots of GE (final concentration in the reaction mixture in the range of $0-1600 \mathrm{nmol}$ ) with $100 \mu \mathrm{L} \mathrm{DPPH}(0.1 \mathrm{mM})$ for 30 minutes. The absorbance was measured at $517 \mathrm{~nm}$ using an enzyme-linked immunosorbent assay plate reader (molecular devices E750). The assay was performed in three replicates for each concentration. The percent DPPH radical scavenged was calculated and expressed as inhibitory concentration $50 \%\left(\mathrm{IC}_{50}\right)$ value. $\mathrm{IC}_{50}$ value was calculated statistically using non-linear regression (dose vs. inhibition).

Modulatory effect of GE against AAPH-induced lipid peroxidation Adult male rats were sacrificed using general anesthesia. Brain and $\mathrm{SN}$ were dissected and brain regions, viz., $\mathrm{Ct}$ and $\mathrm{Cb}$ were separated on ice. $\mathrm{Ct}$ and $\mathrm{Cb}$ were homogenized $(10 \% \mathrm{w} / \mathrm{v})$ separately in icecold phosphate buffered saline $(0.1 \mathrm{M}, \mathrm{pH} 7.4,0.8 \% \mathrm{NaCl})$ centrifuged at $800 \times \mathrm{g}\left(10\right.$ minutes, $\left.4^{\circ} \mathrm{C}\right)$ and the supernatant was collected and freshly used for the in vitro analysis. SN was minced and homogenized

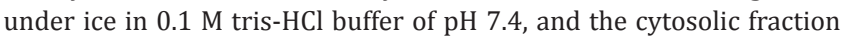
was obtained by centrifuging the samples at 2,000 $\times \mathrm{g}$. Initially, tissue homogenates ( $\mathrm{Ct}, \mathrm{Cb}$, and $\mathrm{SN})$ were incubated $\left(1 \mathrm{hr}, 37^{\circ} \mathrm{C}\right)$ with AAPH $(50,100$, and $200 \mu \mathrm{M})$ in vitro in a final volume of $500 \mu \mathrm{L}$ made up using Krebs-Ringer bicarbonate solution as described earlier and the induction of lipid peroxidation was measured. To assess the potency of GE to inhibit AAPH-induced lipid peroxidation, tissue homogenates challenged with AAPH $(50 \mu \mathrm{M})$ was co-incubated with or without different concentrations $(50,100$, and $200 \mathrm{nmol})$ of GE for $1 \mathrm{hr}$. Separate $\mathrm{GE}$ aliquots were maintained without AAPH to monitor any per se effect. After the incubation period, the extent of formation of lipid peroxides was quantified as levels of malondialdehyde (MDA) by measuring TBA reactive substances.

\section{Cell culture experiments: In vitro model of hyperglycemia in SHSY5Y cells}

\section{Induction of hyperglycemia}

SHSY5Y, a human neuroblastoma cell line, was maintained in Dulbecco's Modified Eagle's Medium media supplemented with 10\% fetal bovine serum and pens trep. The growth conditions include humid atmosphere of $5 \% \mathrm{CO}_{2}$ and $95 \% \mathrm{O}_{2}$ at $37^{\circ} \mathrm{C}$. Cells were plated in 96 well plates at a density of $5 \times 10^{5}$ cells/well. Cells were exposed to different concentrations of glucose (Glc; 25 - $300 \mathrm{mM}$ ) for 24 hrs. MTT [3-(4,5-dimethylthiazol-2-yl)2,5-diphenyltetrazoliumbromide] assay was carried out to determine cell survivability [22]. The yellow tetrazolium MTT gets reduced by metabolically active cells, in part by the action of dehydrogenase enzymes resulting in the formation of purple formazan crystals. These intracellular formazan crystals were then solubilized in SDS-DMF buffer (45\% DMF in distilled water and $10 \%$ SDS, pH 4.7). It was quantified spectrophotometrically at $570 \mathrm{~nm}$, and percent cell survivability was calculated against control (using OD as a measure).

\section{Effect of GE on cell survivability}

A $10 \mathrm{mM}$ stock solution of GE was prepared in 95\% ethanol, while all the working standards were prepared in the culture media. Initially, cells were plated in 96 well plates at a density of $5-10^{5}$ cells/well containing different concentrations (1-100 $\mu \mathrm{M})$ of GE for $24 \mathrm{hrs}$. Cell survival was determined by MTT assay to ascertain the non-toxic concentrations. For further experiments, only selected concentrations were employed, to assess the potential to modulate glucose-induced cellular aberrations and cell death.

\section{Modulatory effects of GE on glucose-induced OS}

SHSY5Y cells were co-exposed to sub-lethal concentrations of GE and $100 \mathrm{mM}$ of glucose ( $\mathrm{IC}_{50}$ concentration) for $24 \mathrm{hrs}$. Cell survival and protection rendered by the active against glucose-induced cell death was determined by MTT assay. For biochemical estimations, experiments were carried out only with a functional concentration of $10 \mu \mathrm{M}$ of GE (i.e., the concentration at which it rendered protection against glucoseinduced cell death). Selected markers for OS (generation of ROS, levels of hydroperoxides (HP) and glutathione [GSH]) were determined post 24 hrs co-exposure.

\section{Modulatory effect of GE on ROS levels}

ROS generation was assayed using 2,7-dichloro-fluorescein diacetate $\left(\mathrm{H}_{2}\right.$ DCFH-DA), a nonpolar compound, which after conversion to a polar derivative by intracellular esterases, rapidly reacts with ROS to form the highly fluorescent compound DCF [23]. Briefly, an aliquot (100 $\mu$ g protein equivalent) was incubated in Locke's buffer (pH 7.4; NaCl: $154 \mathrm{mM}, \mathrm{KCl}$ : $5.6 \mathrm{mM}, \mathrm{NaHCO}_{3}: 3.6 \mathrm{mM}$, HEPES: $5 \mathrm{mM}, \mathrm{CaCl}_{2}: 2 \mathrm{mM}$, and glucose: $10 \mathrm{mM}$ ) containing $\mathrm{H}_{2}$ DCFH-DA ( $5 \mathrm{mM}$ ) for 30 minutes at room temperature. The fluorescent product DCF formed was measured using a spectrofluorimeter with an excitation and emission wavelengths of $480 \mathrm{~nm}$ and $530 \mathrm{~nm}$, respectively. The ROS generation was calculated from a DCF standard curve and expressed as nmol DCF/minutes/mg protein.

\section{Modulatory effect of GE on HP levels}

HP levels were measured according to a previously described method using FOX 1 reagent with minor modifications [24]. An aliquot of cytosolic (or mitochondrial) fraction (100 mg protein) was added to $1 \mathrm{~mL}$ FOX reagent (100 $\mu \mathrm{M}$ xylenol orange; $250 \mu \mathrm{M}$ ammonium ferrous sulfate; $100 \mu \mathrm{M}$ sorbitol; $25 \mathrm{mM} \mathrm{H}_{2} \mathrm{SO}_{4}$ ) and incubated for 30 minutes at room temperature. The color developed was read at $560 \mathrm{~nm}$ in a spectrophotometer. The concentration of HP was calculated using the molar extinction coefficient $\left(\varepsilon=2.2 \times 10^{5} / \mathrm{M} / \mathrm{cm}\right)$ and expressed as nmol $\mathrm{HP} / \mathrm{mg}$ protein.

\section{Modulatory effect of GE on GSH levels}

GSH content was quantified based on a fluorimetric method as described previously using ophthalaldehyde (OPT) [25]. Briefly, an aliquot of the cytosolic fraction was added to formic acid (0.1 M) and centrifuged at $10,000 \times \mathrm{g}$ for 10 minutes. An aliquot of supernatant (deproteinized) was added to tubes containing buffered formaldehyde (1: $4(\mathrm{v} / \mathrm{v}) 37 \%$ formalin: $0.1 \mathrm{M} \mathrm{Na}_{2} \mathrm{HPO}_{4}$ ). Sodium phosphate buffer (0.1 M; pH 8.0; containing $5 \mathrm{mM}$ ethylenediaminetetraacetic acid) was added to each tube followed by OPT $(100 \mu \mathrm{g} / \mathrm{mL})$. Following incubation for 45 minutes at room temperature, the fluorescence was measured (excitation: $345 \mathrm{~nm}$ and emission: $425 \mathrm{~nm}$ ). Concentration of GSH was calculated from a standard curve and values were expressed as nmol/ mg protein.

\section{Modulatory effect of GE on nitrated proteins 3-nitrotyrosine (3-NT)}

The extent of protein nitration was estimated in terms of 3NT employing a slot-blot method. In brief, aliquots (15 $\mu \mathrm{g}$ protein) were spotted in triplicates onto a nitrocellulose membrane. The membranes were treated with a blocking buffer containing $2 \%$ bovine serum albumin in

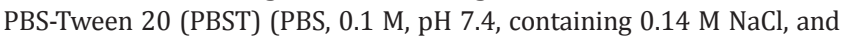
$0.01 \%$ Tween-20) for 60 minutes at room temperature and probed with polyclonal anti-3-NT antibody (1:500; origin- Rabbit; Sigma Chemical Co. USA.) over the next 60 minutes. The membranes were washed repeatedly in PBST and incubated with ALP-conjugated secondary antibody (1:3000; origin-goat; Merck- Bangalore Genei, India) for 60 minutes. The membranes were developed employing BCIP/NBT reaction. To adjust for protein loading, duplicate membranes were also immune-stained with Anti- $\beta$ actin monoclonal antibody (1:2000; Sigma, USA). Optical density on the blots was measured with Image Software (NIH, USA). The extent of protein nitration was expressed as arbitrary units [26].

\section{RESULTS}

\section{Free radical scavenging effect of GE in chemical systems}

\section{DPPH radical scavenging assay}

GE scavenged the free radical DPPH to a yellow colored 1,1-diphenyl-2picrylhydrazyl in a concentration-dependent manner (Fig. 1). The $\mathrm{IC}_{50}$ value was found to be $663 \mathrm{nmol}$ by statistical analysis $\left(\mathrm{R}^{2}=0.9513\right)$. 


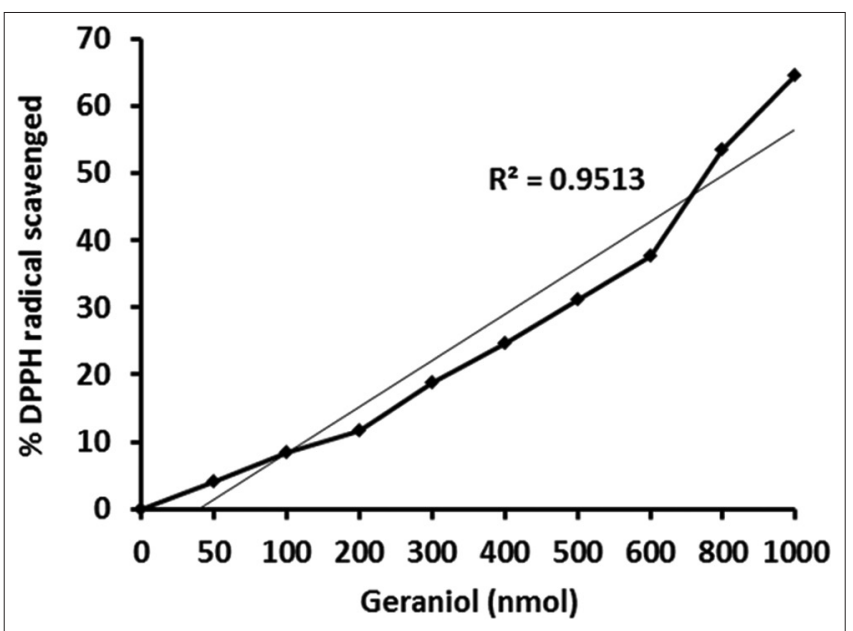

Fig. 1: Free radical scavenging activity of geraniol as determined by diphenyl picrylhydrazyl radical scavenging assay. Values are mean $\pm S E(n=3$ replicates/concentration, from two independent experiments)

Modulatory effect of GE against AAPH-induced lipid peroxidation AAPH exposure resulted in a concentration-dependent increase in lipid peroxidation in different tissues of rat (Data not shown). The elevated levels of MDA (indicative of lipid peroxidation) induced by $50 \mu \mathrm{M}$ AAPH in different tissues were - Ct: $67.7 \pm 6 \%$; $\mathrm{Cb}$ : $90.8 \pm 3 \%$; SN: $69.4 \pm 9 \%$. While GE reduced the extent of lipid peroxidation in $\mathrm{Ct}$ at all concentrations tested, in $\mathrm{Cb}$ and $\mathrm{SN}$ reduction was evident only at 100 nmol concentrations (Fig. 2).

Cell culture experiments: In vitro model of hyperglycemia in SHSY5Y cells

\section{Effect of glucose and GE on cell survivability}

Exposure of SHSY5Y cells to different concentrations of glucose resulted in a concentration-dependent cell death as evidenced by MTT reduction assay. Based on these previous preliminary studies, for studying the modulatory potency of $\mathrm{GE}$, glucose concentration of $100 \mathrm{mM}\left(\mathrm{IC}_{50}\right)$ was used [27]. Further, exposure of cells to GE per se did not cause any cell death in the concentration range of 5-50 mM. Hence, the concentrations used for studying the modulatory potency of GE against hyperglycemia were in the range of 5-50 $\mu \mathrm{M}$. GE offered protection $(\mathrm{p} \leq 0.05)$ only at the concentration of $10 \mu \mathrm{M}$ (Fig. 3).

\section{Modulatory effects of GE on glucose-induced OS}

Glucose exposure caused significant induction of OS as evidenced by elevation in ROS (20\%) and HP (50\%) levels as well as depletion in GSH levels (35\%) (Table 1). GE exposed cells exhibited a significant reduction in the endogenous levels of ROS (37\%) and HP (27\%). While ROS levels were reduced marginally by GE when co-exposed with glucose, the HP levels were normalized (Fig. 4a and b). While no alteration in the endogenous levels of GSH/GSSG with GE exposure was evident, on co-exposure with glucose, the GSH levels were increased two folds (Table 1).

\section{Modulatory effects of GE on 3-NT levels}

Glucose exposure to SHSY5Y cells resulted in a marked increase (46\%) in 3-NT levels. While GE reduced the endogenous levels moderately, a marked diminution (about three-fold against glucose per se) occurred when co-exposed with glucose (Fig. 5).

\section{DISCUSSION}

The complexity of biochemical pathways in central nervous system and peripheral nervous system in NDD is not fully understood. Nevertheless, OS and inflammatory reactions play a central role in degenerative processes. This necessitates to target the increase in OS by
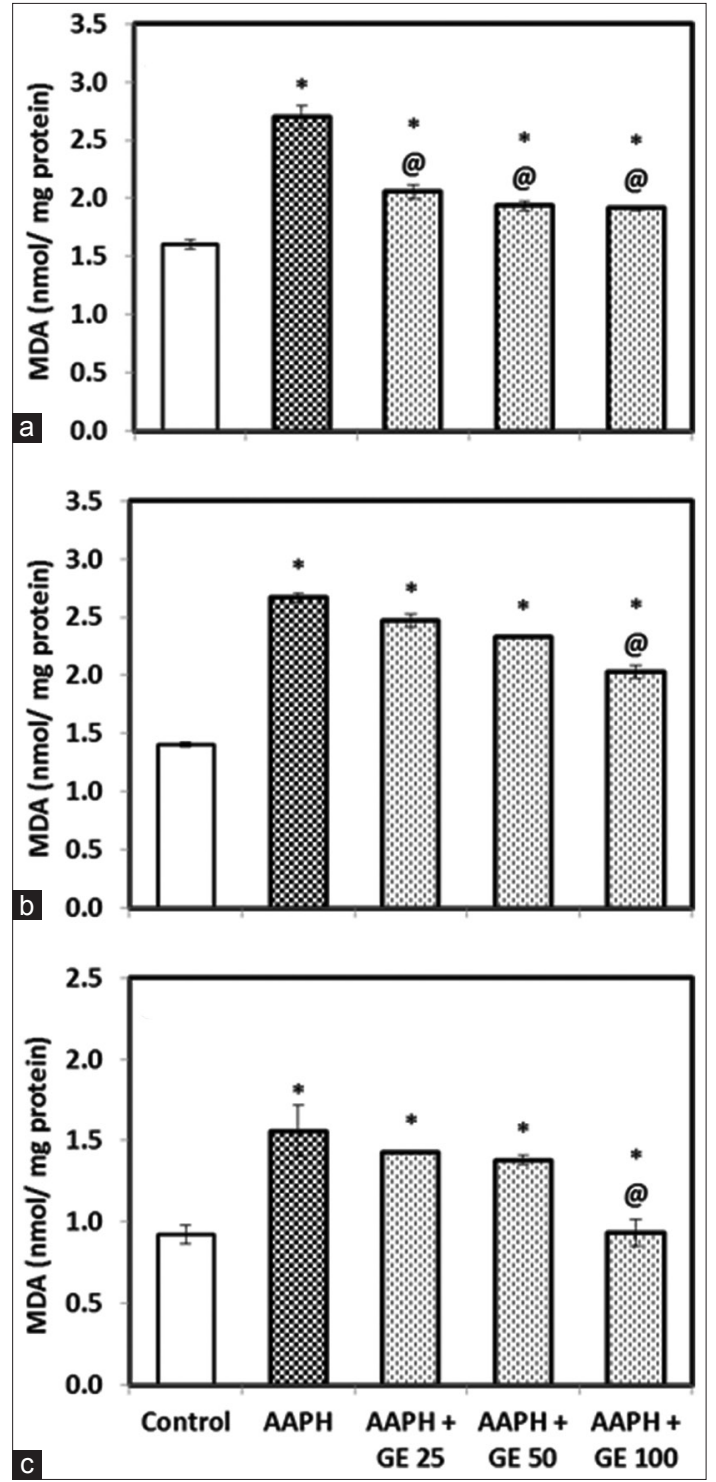

Fig. 2: Effect of geraniol on 2,2'-azobis(2-methylpropionamidine) (APPH) induced lipid peroxidation in cortex (a), cerebellum (b) and sciatic nerve (c) of male Wistar rats. Values are mean $\pm S E$ ( $n=3$ replicates/concentration, from two independent experiments). Data analyzed by one-way analysis of variance followed by Tukey's test for comparison of means. *Significant against control, @significant against AAPH at p<0.05

supplementing with antioxidants and/or to fortify antioxidant defense system to keep a check on the progression of different degenerative pathways. This is true of neurodegeneration seen in the case of $A D$, PD or DN. Several labs including ours have been investigating the possibilities of employing plant based actives against NDD [9-11,28]. The mechanisms through which supplementation of such bioactives may diminish free radical-related diseases like NDD is associated to their multiple biological abilities. This includes their ability to reduce the levels of or the formation of ROS and RNS, concomitantly upregulating vitagenes, such as members of the heat shock protein (Hsp) family, heme oxygenase-1, and Hsp70 [8,26].

GE has been reported as an effective antioxidant and antiinflammatory agent in different experimental models from our lab and others [16-19,21,29]. Recently, the antioxidant activity of GE along with other actives has been published with respect to DPPH free radical scavenging activity and superoxide dismutase activity [30]. However, 
this study was planned and executed bearing in mind our results in different experimental models of neurodegeneration [16,21]. These studies emphasized the modulatory potency of oral supplementation of GE against acrylamide induced neurotoxicity and DN. In both the in vivo studies, it was evident that GE supplementation could interfere with OS by altering the levels of oxidative markers such as ROS, hydrogen peroxides, nitrites and protein carbonyls in brain regions, and SN. Hence in this study, first the DPPH free radical scavenging capacity was determined and was found to be concentration dependent (correlation: $\left.\mathrm{R}^{2}=0.9513\right)$. Further, the homogenates of $\mathrm{Ct}, \mathrm{Cb}$, and $\mathrm{SN}$ were challenged with AAPH leading to the induction of lipid peroxidation in these high lipid containing nervous tissues. This mimics the condition of high lipid peroxidation levels noted in NDD. The modulatory potential of GE against AAPH induced lipid peroxidation was clearly evident by the reduced levels of MDA in all tissue homogenates tested. This emphasizes the role of GE to reduce lipid peroxidation in the in vivo models studied earlier.

Further, experiments were carried out in media containing high levels of glucose in the SHSY5Y undifferentiated cells simulating diabetic condition. In the case of neuronal cells the glucose uptake is facilitated diffusion, and hence whenever the blood glucose levels are high as is the case of diabetes, the neuronal glucose level is elevated [31,32]. Hyperglycemia leads to the activation of various glucose-mediated pathways such as polyol pathway, AGE formation pathway, activation of

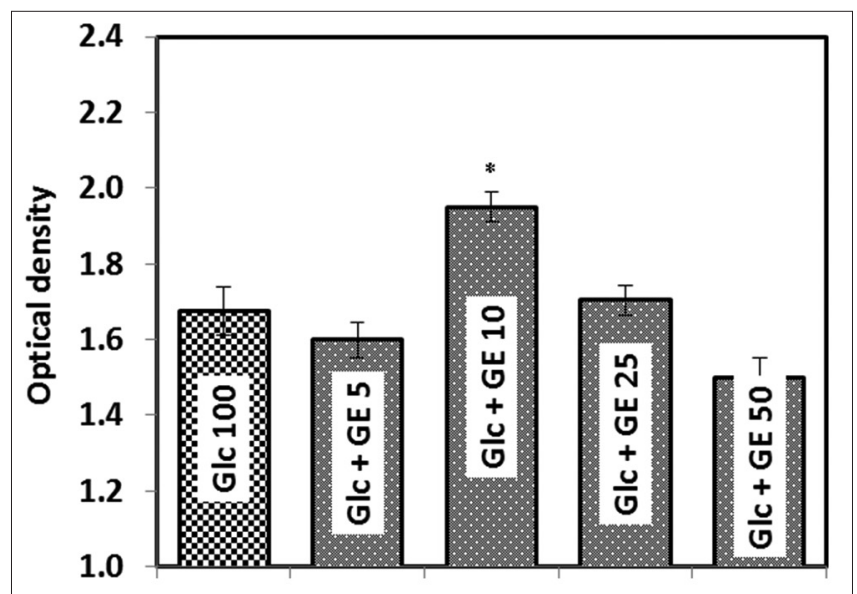

Fig. 3: Modulatory effect of geraniol (GE 5, 10 and $20 \mu \mathrm{M}$ ) on co-exposure with glucose (Glc, $100 \mathrm{mM}$ ) in SHSY5Y cells for 24 hrs; values are mean \pm SE ( 3 sets of experiments). Data analyzed by one-way analysis of variance followed by Tukey's test for comparison of means. *Significant $\mathbf{p}<0.05$ against glucose (Glc, $100 \mathrm{mM}$ ) protein kinase A and hexosamine monophosphate pathway ultimately culminating to the development of OS, inflammatory reactions and apoptotic process [2,33]. In SHSY5Y cells, hyperglycemic condition induced cell death and elevation in OS markers such as ROS, hydrogen peroxides, and 3-nitrotyrosine $[27,34]$. GE co-exposure with the $\mathrm{IC}_{50}$ level of glucose rescued the cells from death. Further, enhanced levels of ROS and hydrogen peroxide were significantly reduced with GE clearly suggesting its ability to interfere OS related mechanism under high glucose levels. Interestingly, GE also enhanced the depleted levels of GSH with concomitant change (about 20\%) in the levels of GSSG (Data not shown). This is in line with our previous findings in streptozotocin model of diabetes in rat where GE significantly altered the oxidative markers in different regions of the brain and SN [16]. Further, antioxidant effect is a key feature which is being investigated by several diabetes researchers and hunt for novel phytochemicals alone or in the form of extracts is going on worldwide [32,35].

\section{CONCLUSION}

In this study, the in vitro analysis employing various models exhibited the antioxidant activity of GE. These findings and studies from other labs further emphasizes the potential of GE against free radical mediated deleterious effects under various disease conditions like NDD $[18,31,36]$. GE is hence proposed to be employed as an adjuvant in the management or prevention of neurodegenerative processes involved in NDD like DN.

\section{ACKNOWLEDGMENT}

The authors wish to thank the Director, CSIR-Central Food Technological Research Institute, Mysuru, for his keen interest in this area of research and Dr. MMS Bharath, Department of Neurochemistry, National Institute of Mental Health and Neurosciences for his kind support. Furthermore, the grant from the Department of Science and Technology (Women Scientist Scheme WOS - A) New Delhi, Government of India (Ref: SR/WOS-A/LS-201/2008) to the first author is greatly acknowledged.

Table 1: Modulatory effect of geraniol (GE $10 \mu \mathrm{M}$ ) on reduced GSH in SHSY5Y cells co-exposed with glucose (Glc, $100 \mathrm{mM}$ ) for $24 \mathrm{hrs}$

\begin{tabular}{ll}
\hline Group & GSH (nmol/ mg protein) \\
\hline Control & $22.28 \pm 0.7$ \\
GE $10 \mu \mathrm{M}$ & $22.52 \pm 1.2$ \\
Glc $100 \mathrm{mM}$ & $14.92 \pm 0.8^{*}$ \\
Glc + GE $10 \mu \mathrm{M}$ & $29.16 \pm 0.3^{\#}$ \\
\hline
\end{tabular}

GSH: Glutathione, Data analyzed by one-way analysis of variance followed by Tukey's test for comparison of means. * *ignificant against control, \#against glucose (Glc, 100mM), p $\leq 0.001$
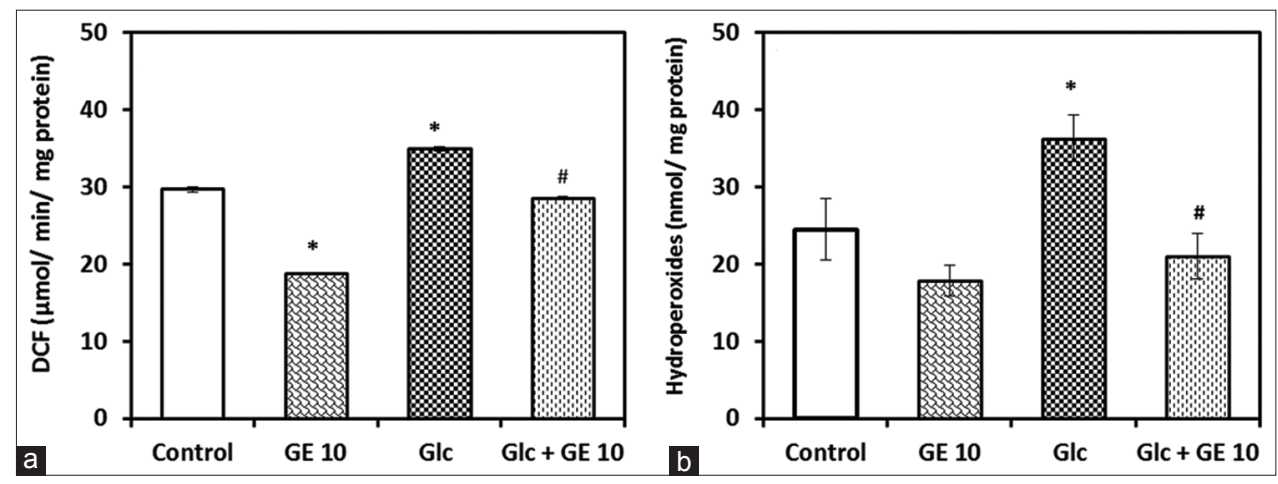

Fig. 4: Modulatory effect of geraniol (GE $10 \mu \mathrm{M}$ ) on reactive oxygen species (a) and hydroperoxides (b) when co-exposed with glucose (Glc, $100 \mathrm{mM}$ ) in SHSY5Y cells for $24 \mathrm{hrs}$. Values are mean \pm SE (3 sets of experiments). Data analyzed by one-way analysis of variance followed by Tukey's test for comparison of means. *Significant $(p<0.05)$ against control and \#significant $(p<0.05)$ against glucose (Glc, $100 \mathrm{mM}$ ) 


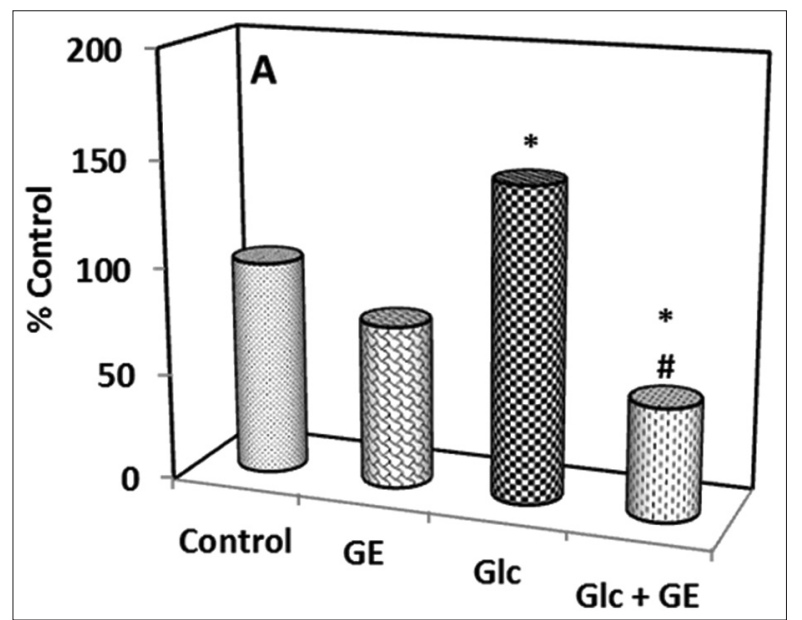

Fig. 5: Modulatory effect of geraniol (GE, $10 \mu \mathrm{M})$ on the levels of 3-nitrotyrosine in SHSY5Y cells co-exposed with glucose (Glc, $100 \mathrm{mM}$ ) for $24 \mathrm{hrs}$ as evidenced by slot blot analysis. Values are mean \pm SE ( 3 sets of experiments). Data analyzed by one-way analysis of variance followed by Tukey's test for comparison of means. *Significant $(\mathbf{p}<0.05)$ against Control and \# significant $(p<0.05)$ against glucose $(\mathrm{Glc}, 100 \mathrm{mM})$

\section{REFERENCES}

1. Halliwell B. Oxidative stress and neurodegeneration: Where are we now? J Neurochem 2006;97(6):1634-58.

2. Obrosova IG. Diabetes and the peripheral. Biochem Biophys Acta 2009;10:931-40.

3. Kell DB. Towards a unifying, systems biology understanding of largescale cellular death and destruction caused by poorly liganded iron: Parkinson's, Huntington's, Alzheimer's, prions, bactericides, chemical toxicology and others as examples. Arch Toxicol 2010;84(11):825-89.

4. Kumar A, Negi G, Sharma SS. Suppression of NFkB and NFkB regulated oxidative stress and neuroinflammation by BAY11-7082 (IJB phosphorylation inhibitor) in experimental diabetic neuropathy. Biochimie 2012;94:1158-65.

5. Yorek MA. Treatment of diabetic neuropathy with baicalein: Intervention at multiple sites. Exp Neurol 2011;232(2):105-9.

6. Singh M, Arseneault M, Sanderson T, Murthy V, Ramassamy C. Challenges for research on polyphenols from foods in Alzheimer's disease: Bioavailability, metabolism, and cellular and molecular mechanisms. J Agric Food Chem 2008;56(3):4855-73.

7. Le Bars PL, Katz MM, Berman N, Itil TM, Freedman AM, Schatzberg AF. A placebo-controlled, double-blind, randomized trial of an extract of Ginkgo biloba for dementia. North American EGB Study Group. JAMA 1997;278(16):1327-32.

8. Calabrese V, Cornelius C, Trovato A, Cavallaro M, Mancuso C, Di Rienzo L, et al. The hormetic role of dietary antioxidants in free radicalrelated diseases. Curr Pharm Des 2010;16(7):877-83.

9. Prasad SN, Muralidhara. Neuroprotective efficacy of eugenol and isoeugenol in acrylamide-induced neuropathy in rats: Behavioral and biochemical evidence. Neurochem Res 2013;38(2):330-45.

10. Chongtham A, Agrawal N. Curcumin modulates cell death and is protective in Huntington's disease model. Sci Rep 2016;6:18736.

11. Pérez-Hernández J, Zaldívar-Machorro VJ, Villanueva-Porras D, Vega-Ávila E, ChavarríaA.Apotential alternative against neurodegenerative diseases: Phytodrugs. Oxid Med Cell Longev 2016;2016:8378613.

12. Madankumar A, Jayakumar S, Gokuladhas K, Rajan B, Raghunandhakumar S, Asokkumar S, et al. Geraniol modulates tongue and hepatic phase I and phase II conjugation activities and may contribute directly to the chemopreventive activity against experimental oral carcinogenesis. Eur J Pharmacol 2013;705(1-3):148-55.

13. Lapczynski A, Bhatia SP, Foxenberg RJ, Letizia CS, Api AM. Fragrance material review on geraniol. Food Chem Toxicol 2008;46 Suppl 11:S160-70.
14. Chen W, Viljoen AM. Geraniol - A review of a commercially important fragrance material. S Afr J Bot 2010;76:643-51.

15. Singh BK, Tripathi M, Chaudhari BP, Pandey PK, Kakkar P. Natural terpenes prevent mitochondrial dysfunction, oxidative stress and release of apoptotic proteins during nimesulide-hepatotoxicity in rats. PLoS One 2012;7(4):e34200.

16. Prasad SN, Muralidhara. Protective effects of geraniol (a monoterpene) in a diabetic neuropathy rat model: Attenuation of behavioral impairments and biochemical perturbations. J Neurosci Res 2014;92(9):1205-16.

17. Wang J, Su B, Zhu H, Chen C, Zhao G. Protective effect of geraniol inhibits inflammatory response, oxidative stress and apoptosis in traumatic injury of the spinal cord through modulation of NF-KB and p38 MAPK. Exp Ther Med 2016;12(6):3607-13.

18. Kumar MA, Devaki T. Geraniol, a component of essential oils - A review of its pharmacological properties. Int J Pharm Pharm Sci 2015;7:67-70.

19. Tiwari M, Kakkar P. Plant derived antioxidants - Geraniol and camphene protect rat aveolar macrophages against t-BHP induced oxidative stress. Toxicol in vitro 2009;23:295-301.

20. Halliwell B. Are polyphenols antioxidants or pro-oxidants? What do we learn from cell culture and in vivo studies? Arch Biochem Biophys 2008;476(2):107-12.

21. Prasad SN, Muralidhara. Mitigation of acrylamide-induced behavioral deficits, oxidative impairments and neurotoxicity by oral supplements of geraniol (a monoterpene) in a rat model. Chem Biol Interact 2014;223:27-37.

22. Russo VC, Higgins S, Werther GA, Cameron FJ. Effects of fluctuating glucose levels on neuronal cells in vitro. Neurochem Res 2012;37(8):1768-82.

23. Chandrashekar KN, Muralidhara. Oxidative alterations induced by D-aspartic acid in prepubertal rat testis in vitro: A mechanistic study. Theriogenology 2008;70(1):97-104.

24. Wolff SP. Ferrous ion oxidation in the presence of ferric ion indicator xylenol orange for measurement of hydrogen peroxides. Med Enzymol 1994;233:182-9.

25. Mokrasch LC, Teschke EJ. Glutathione content cultured cells and rodent brain regions: Specific fluorimetric assay. Anal Biochem 1984;140:506-9.

26. Chandran G, Muralidhara. Neuroprotective effect of aqueous extract of Selaginella delicatula as evidenced by abrogation of rotenone-induced motor deficits, oxidative dysfunctions, and neurotoxicity in mice. Cell Mol Neurobiol 2013;33(7):929-42.

27. Prasad SN, Bharath MM, Muralidhara. Neurorestorative effects of eugenol, a spice bioactive: Evidence in cell model and its efficacy as an intervention molecule to abrogate brain oxidative dysfunctions in the streptozotocin diabetic rat. Neurochem Int 2016;95:24-36.

28. Hosamani R, Muralidhara. Prophylactic treatment with Bacopa monnieri leaf powder mitigates paraquat-induced oxidative perturbations and lethality in Drosophila melanogaster. Indian J Biochem Biophys 2010;47(2):75-82

29. Ahmad ST, Arjumand W, Seth A, Nafees S, Rashid S, Ali N, et al. Preclinical renal cancer chemopreventive efficacy of geraniol by modulation of multiple molecular pathways. Toxicology 2011;290(1):69-81.

30. Farhath MS, Vijaya PP, Vimal M. Antioxidant activity of geraniol, geranial acetate, gingerol and eugenol. Res Pharm 2013;3:1-6.

31. Brownlee M. The pathobiology of diabetic complications: A unifying mechanism. Diabetes 2005;54(6):1615-25.

32. Pal S, Gautam S, Mishra A, Srivastava AK. Antihyperglycemic and antidyslipidemic potential of Ipomoea batatas leaves in validated diabetic model. Int J Pharm Pharm Sci 2015;7:176-86.

33. Vincent AM, Russell JW, Low P, Feldman EL. Oxidative stress in the pathogenesis of diabetic neuropathy. Endocr Rev 2004;25(4):612-28.

34. Hsu YY, Tseng YT, Lo YC. Berberine, a natural antidiabetes drug, attenuates glucose neurotoxicity and promotes Nrf2-related neurite outgrowth. Toxicol Appl Pharmacol 2013;272(3):787-96.

35. Laaboudi W, Ghanam J, Ghoumari O, Sounni F. Hypoglycemic and hypolipidemic effects of phenolic olive tree extract in streptozotocin diabetic rats. Int J Pharm Pharm Sci 2016;8:287-91.

36. Prasad SN, Muralidhara. Neuroprotective effect of geraniol and curcumin in an acrylamide model of neurotoxicity in Drosophila melanogaster: Relevance to neuropathy. J Insect Physiol 2014;60:7-16. 